\title{
Climate risk management requires explicit representation of societal trade-offs
}

\author{
Gregory Garner $^{1} \cdot$ Patrick Reed $^{2} \cdot$ Klaus Keller $^{1,3,4}$
}

Received: 23 October 2015 / Accepted: 13 January 2016 / Published online: 22 January 2016

C The Author(s) 2016. This article is published with open access at Springerlink.com

\begin{abstract}
Strategies for managing climate-change risks impact diverse stakeholder groups that possess potentially conflicting preferences. Basic physics and economics suggest that reconciling all of these preference conflicts may not be possible. Moreover, different climate risk management strategies can yield diverse and potentially severe impacts across different global stakeholders. These preference conflicts and their uncertain impacts require an explicit understanding of the trade-offs that emerge across different risk management strategies. Traditionally, integrated assessment models (IAMs) typically aggregate the stakeholders' preferences across the entire globe into a single, a priori defined utility function. This framing hides climate risk management trade-offs as well as the inherent stakeholder compromises implicit to the resulting single "optimal" expected utility solution. Here, we analyze a simple IAM to quantify and visualize the multidimensional trade-offs among four objectives representing global concerns: (i) global economic productivity, (ii) reliable temperature stabilization, (iii) climate damages, and (iv) abatement costs. We quantify and visualize the trade-offs across these objectives and demonstrate how a traditional optimal expected utility policy implicitly eliminates many relevant policy pathways. Explicit trade-off analysis provides a richer context for exploring conflicting global policy preferences and clarifies the implications of alternative climate risk mitigation policies to better inform negotiated compromises.
\end{abstract}

Electronic supplementary material The online version of this article (doi:10.1007/s10584-016-1607-3) contains supplementary material, which is available to authorized users.

Gregory Garner

ggg121@psu.edu

1 Earth and Environmental Systems Institute, The Pennsylvania State University, University Park, Pennsylvania 16803, USA

2 School of Civil and Environmental Engineering, Cornell University, Ithaca, New York 14853, USA

3 Department of Geosciences, The Pennsylvania State University, University Park, Pennsylvania 16803, USA

4 Department of Engineering and Public Policy, Carnegie Mellon University, Pittsburgh, Pennsylvania 15213, USA 
The goal of the internationally ratified Framework Convention on Climate Change is to stabilize the level of greenhouse gas concentrations in the atmosphere to a level that "would prevent dangerous anthropogenic interference with the climate system" in a way that "enable[s] economic development to proceed in a sustainable manner" (United Nations 1992). As described, this agreement seeks to balance the policy trade-offs that emerge when reducing greenhouse gas emissions while seeking to maintain global economic productivity. Integrated assessment models (IAMs) represent one class of tools that are being used to evaluate the balance between climate risk mitigation and global economic welfare. IAMs of climate change and the economy typically use utility-based abstractions of global welfare preferences to analyze and select optimal climate change mitigation strategies (Weyant et al. 1996). Framing the problem in terms of utility maximization aggregates the values and preferences of diverse stakeholder groups into a single value function that is used to rank alternative mitigation strategies. Simply maximizing the expectation of utility derives the optimal decision for a single hypothetical rational agent. This approach has provided important insights (e.g., Nordhaus 2013, McInerney et al. 2012) and is based on simple axioms (Bentham 1879, Ramsey 1931, Von Neumann and Morgenstern 1945); however, maximizing the expected utility can have poor descriptive power for real decision making (Ellsberg 1961; Banzhaf 2009).

We use the Dynamic Integrated Climate-Economy model (DICE) to illustrate this commensuration conundrum (Nordhaus 2013). DICE is one of three IAMs used by the United States Environmental Protection Agency in determining the social cost of carbon, a quantity used in cost-benefit analyses of climate-change mitigation and adaptation strategies (Greenstone et al. 2013). The standard approach in DICE is to derive an optimal strategy by maximizing the discounted expected utility of per capita consumption. When using this approach, it is important to understand the implicit compromises made among critical stakeholder preferences. To do so, we expand the problem formulation in DICE to consider four objectives that approximate key stakeholder concerns: (i) expected utility maximization (EUM) where the primary focus of the stakeholder is that the benefits justify the costs (U.S. Office of Management and Budget 2003), (ii) maximize the reliability of limiting global mean temperature rise to two degrees Celsius (Held et al. 2009) (REL2C) for those concerned about, "dangerous anthropogenic interference with the Earth System" (United Nations 1992), (iii) minimize the expected discounted climate damages (DAM) to approximate the preferences of those concerned about vulnerable systems (McGranahan et al. 2007), and (iv) minimize the expected cost of a carbon-abatement policy (ABATE) to represent those concerned with the costs of mitigating climate change (Heggelund 2007). These objectives abstract highly diverse stakeholder values and potential preferences that are vital to understanding the broader contexts climate risk decisions (Gupta et al. 2007).

Conflicting stakeholder preferences are often abstracted using a summation of alternative mathematical forms as well as weightings of the abatement costs and damage functions (e.g., Kolstad and Toman 2001, Keller et al. 2000, Keller et al. 2005, McInerney et al., 2012). For example, a stakeholder concerned with reliably keeping the global mean temperature increase below two degrees Celsius may define a damage function with extraordinary costs when the temperature exceeds the threshold. Conversely, a stakeholder concerned about abatement costs may choose a steeply-increasing abatement cost function. Drouet et al. (2015) provides a recent illustration of this general approach in representing different stakeholder values and risk preferences. These aggregated approximations for conflicting stakeholder preferences can provide useful insights, but they face several challenges. For one, this approach hides the 
weighting of societal preferences in an a priori choice of the cost functions and aggregates into a single objective, thus the tradeoff among the societal preferences becomes muddled and difficult to analyze. Moreover, the complex group-decision-making context merits more explicit understanding of how policy pathways impact tradeoffs in societal values (National Research Council 2009).

While the four objectives explored in this study are a small subset of the possible stakeholder preferences, but they provide sufficient coverage to illustrate the implicit trade-offs of the EUM formulation. For easier interpretation, we re-cast expected utility in terms of balanced growth equivalent (Anthoff and Tol 2009), which expresses the change in consumption stream from that of a no-climate-damages case for a given policy (Fig. 1a, purple box EUM solution). A sole focus on achieving optimal expected utility implicitly commits us to a non-negligible degree of future climate damages, which may not be tolerable by certain stakeholders (Fig. 2, purple box/line). The a priori definition of utility as the single metric of concern can produce decision myopia.

Expanding the problem formulation by considering a broader suite of objectives can reveal decision relevant trade-offs and reduce decision myopia. For example, adding the reliability of the two degrees Celsius stabilization goal (REL2C) recasts the context and implications of the EUM solution. The two-objective sub-space of the full four objective problem shown highlights a strong trade-off between EUM and REL2C (Fig. 1b). The ideal solution would maximize both EUM and REL2C. This ideal solution, however, is infeasible given the coupled geophysical and economic constraints of the model. The boundary between the feasible and infeasible solutions is the Pareto-front or "trade-off curve" (black line). Solutions on the trade-off curve cannot be improved upon in the underlying model with respect to one objective without degrading the other objective. The trade-off curve is bounded by solutions that optimize each of the objectives separately (blue box - EUM, green box - REL2C) and spans the entire range of possible REL2C values $(0 \%-100 \%)$ (Fig. 1b). Given the strong tension between these two objectives, it would be difficult to argue that the preference represented by the REL2C objective is captured in the EUM problem formulation or appropriated any weight in the a priori definition of utility. Unless explicitly represented in a multi-objective problem formulation, this trade-off would remain hidden, presenting a case of myopia.

Increasing the number of objectives visualized to cover the full four-objective formulation (Fig. 1c) clarifies the trade-offs that emerge when moving away from the extreme solutions that optimize each individual preference perspective. The primary axes in this visualization are the REL2C, DAM, and ABATE objectives while the color gradient represents the EUM objective. The optimal single-objective solutions for the DAM (orange box) and ABATE (cyan box) objectives occupy the extreme ends of the newly introduced visualized trade-offs. While each of these boxes represents an optimal solution for a single objective, they do not provide a compromise over preferences. In a decision-making scenario, the decision makers would either be implicitly (e.g., Fig. 1a) or explicitly (e.g., Fig. 1c) trading off performance among the objectives (Herman et al. 2014). The four-objective visual trade-off analysis provides a richer context for explicitly selecting and understanding compromise climate mitigation solutions. As an illustration of potential compromises, we have selected two solutions as if decision makers selected them for further discussion (Fig. 1c, purple and cyan circles). Although these solutions do not represent actual real-world elicited choices, they are helpful in illustrating how explicit trade-off analyses can aid in understanding the implications of negotiated compromises. 

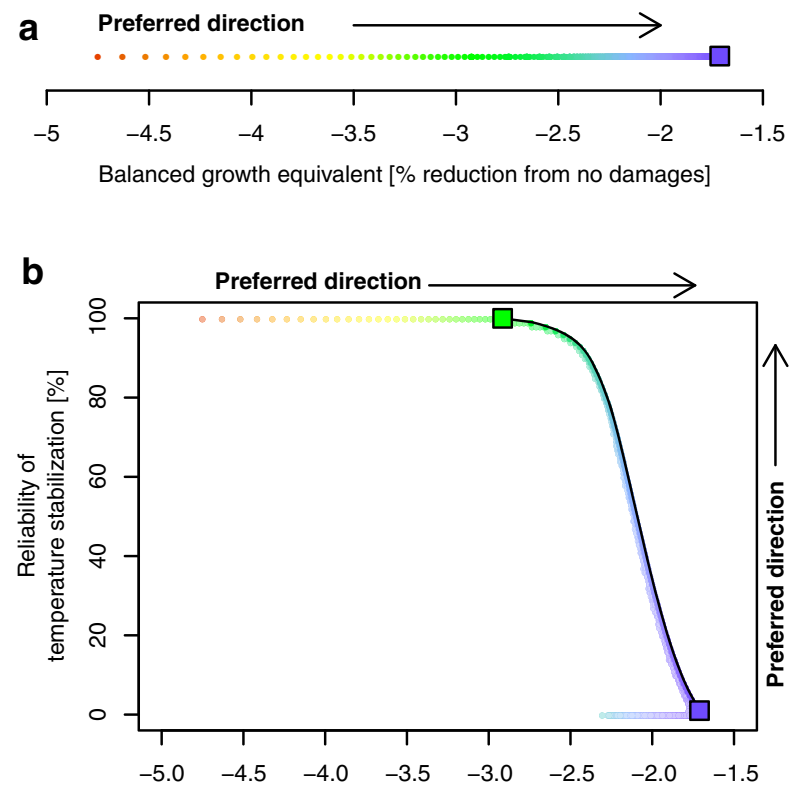

Balanced growth equivalent [\% reduction from no damages]

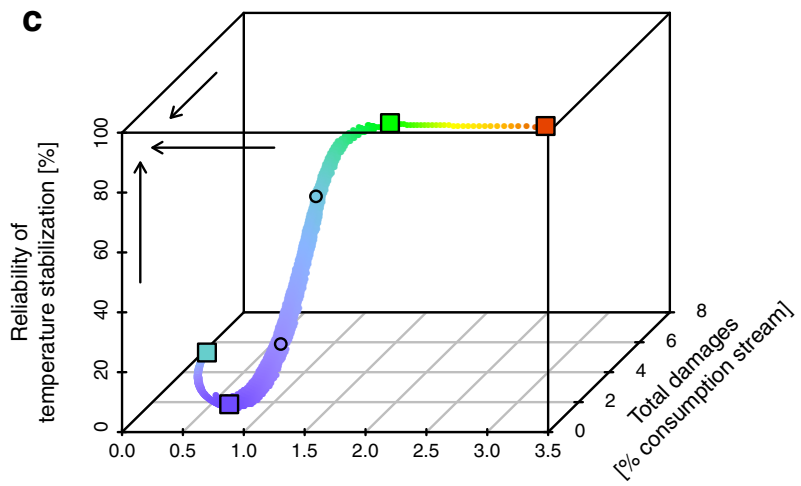

Total abatement cost [\% consumption stream]

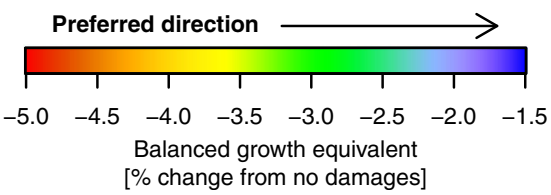

Fig. 1 DICE problem formulation expansion. We expand the DICE problem formulation from (A) singleobjective EUM to (B) a two-objective formulation trading off between EUM and REL2C and finally to (C) the four-objective problem formulation that includes ABATE and DAM. Squares represent the optimal solution for each of the single objectives and circles are illustrative compromise solutions. Arrows indicate the preferred direction of the associated objective (i.e., increasing reliability of temperature stabilization or decreasing total damages)

Intermediate or compromise solutions chosen from the trade-off curve may provide satisficing outcomes across all stakeholder preferences whereas the single objective optimal 


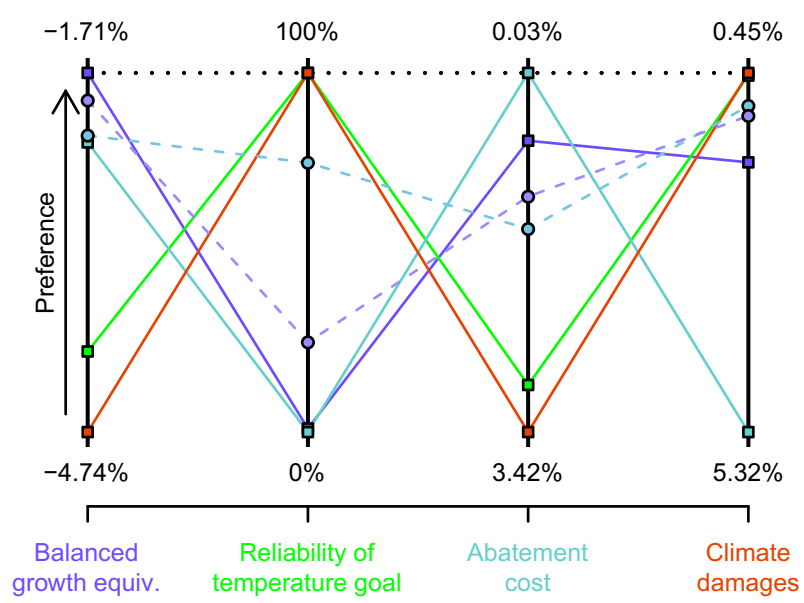

Fig. 2 Parallel axis plot of the four single-objective optimal solutions and two compromise solutions. Each line represents how a single solution performs in each of the objectives. The arrow on the left indicates the direction of preference. The ideal solution would be one that produces a straight line across the top of the plot. Lines that slope between objectives indicate a conflict between the objectives such that in order to perform well in one objective, the solution must deteriorate in one or more other objectives. Solid lines with square points represent the optimal single-objective solutions (EUM - purple, REL2C - green, ABATE - cyan, DAM - orange). Dashed lines with circle points are the illustrative compromise solutions. The ranges of values achieved for each objective in the multi-objective analysis are provided along the top and bottom of the plot

solutions often represent extreme perspectives (Simon 1955; Yohe 2009) (Fig. 2). The EUM solution (purple solid line in Fig. 2) prioritizes a balance between abatement costs and climate damages, but at the expense of reliably achieving temperature stabilization. In fact, the application of the discount rate in the DICE model influences the EUM solution to prefer present day consumption over avoiding the discounted effects of future climate damages, resulting in a climate risk management strategy that most closely mimics a sole focus on abatement cost (see the cyan ABATE solution in Fig. 2). The steep slopes between objectives in Fig. 2 indicate a strong trade-offs across objective pairs and highlight that finding a single ideal solution that is optimal across all stakeholder preferences is impossible in the considered model. The 4-objective tradeoffs provide a richer context for understanding potential climate risk management compromises as well as their implications (Fig. 2, dashed lines). For example, the cyan compromise solution demonstrates how increasing the reliability of stabilizing atmospheric temperatures drastically decreases future climate damages by increasing abatement investments to a level that yields a relatively modest reduction in global growth equivalent (i.e., EUM).

More broadly, the climate risk mitigation solutions highlighted in our analysis (Fig. 2) provide important insights with regard to the dependency of reliable stabilization on an immediate, and sustained increase in global abatement efforts. The EUM solution results in a relatively slow increase in abatement over time (purple line, Fig. 3). The exact numerical results are, of course, subject to many caveats (discussed below). Nonetheless, the general results highlight an important concept. The EUM solution commits us to a path with potentially irreversible consequences. For example, the relatively limited action in the EUM solution would render other important objectives, such as REL2C, unachievable (Figs. 2 and 3). When accounting for policy inertia (Grübler et al. 1999; Keller et al. 2008), the high levels of performance for DAM and REL2C available in the unconstrained formulation are already 


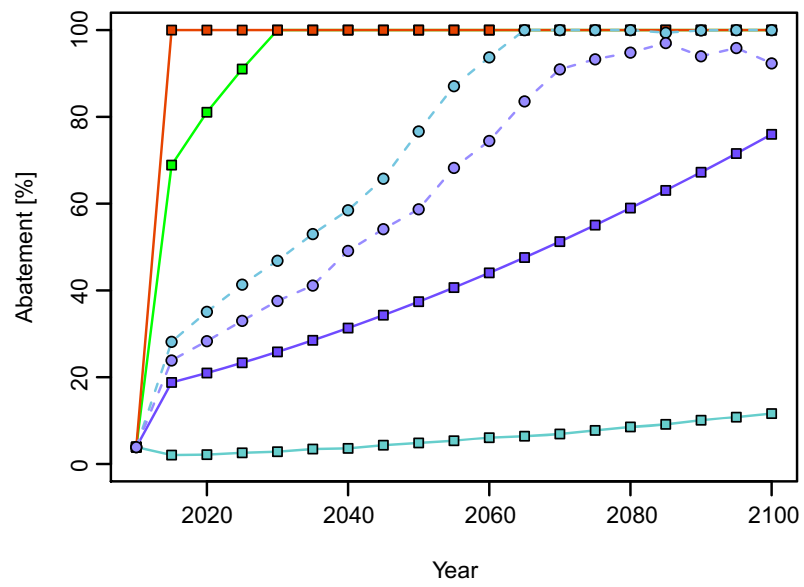

Fig. 3 Optimal and compromise abatement strategies. Abatement strategies associated with the four singleobjective optimal solutions (solid lines with boxes: EUM - purple, REL2C - green, ABATE - cyan, DAM orange) and two illustrative compromise solutions (dashed lines with circles). The abatement strategy is expressed as an emissions control rate of greenhouse gases where $100 \%$ abatement means a complete reduction of emissions. The time-horizon for the analysis is year 2300, but results are shown out to the end of this century

infeasible (Fig. 4). The myopia of present climate-negotiations is effectively removing stakeholders from the decision, which highlights the need for broadening the dialogue on the ethics of climate risk management decisions.

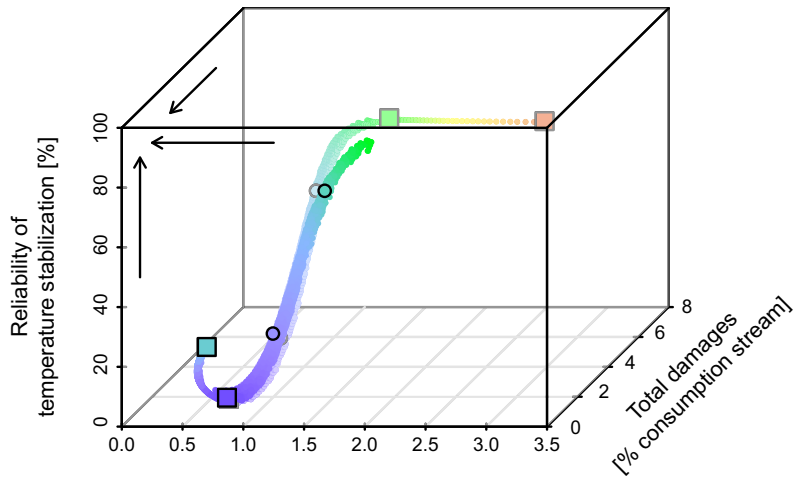

Total abatement cost [\% consumption stream]

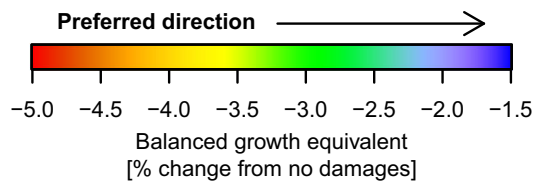

Fig. 4 Effects of policy inertia on the four-objective Pareto-optimal solution set. Akin to Fig. 1c, the squares represent the single objective optimal solutions and the circle represents the illustrative compromise solution. The brushed solutions are those from the unconstrained problem formulation, while the opaque solutions are those from the formulation with a $20 \%$ per-five-year control policy constraint. The control policy inertia constraint is generous relative to those published in the literature (Grübler et al. 1999; Keller et al. 2008), yet the REL2C and DAM optimal solutions from Fig. 1c are infeasible under this constraint. A more strict inertia constraint would remove even more solutions, making it difficult to find satisficing solutions across stakeholders' preferences 
Our results use a simple and transparent model to illustrate the power of a multi-objective problem formulation for understanding the implicit choices and trade-offs that are embedded in the standard EUM approach to analyzing climate-change risk management decisions. This simplicity, however, results in several caveats. For example, the DICE model considers only globally aggregated outcomes and is thus silent on critical questions surrounding intragenerational justice (Rawls 2001). Furthermore, our analysis considers a rather shallow sample of the deep uncertainties (e.g., Butler et al. 2014, Hall et al. 2012). As a last example, our considered objective space is a small subset of the diverse values and objectives relevant to the design of climate change strategies.

Despite these caveats, we show that the standard problem formulation hinges on strong assumptions about the relevance of stakeholder preferences. Specifically, the standard formulation is vulnerable to questionable assumptions in how it exploits an a priori-weighted representation of the potentially conflicting preferences of multiple stakeholders. Explicitly evaluating the higher-dimensional trade-offs reduces this vulnerability and provides richer contextual support for choosing negotiated climate risk mitigation strategies. We demonstrate how concessions in expected utility can lead to large gains in Earth-system related objectives and the externalities of climate change on vulnerable populations. We also show that committing to an abatement policy that maximizes global utility could possibly remove relevant stakeholder preferences from the problem, raising concerns about the ethics of climate-related decision-making. The new formulation can improve the ability of IAMs to provide decision support through an a posteriori assessment of performance criteria over the Pareto-optimal solution set.

\section{Methods}

\subsection{Porting DICE to $\mathrm{C} / \mathrm{C}++$}

We ported the GAMS version of the DICE 2013R model (DICE2013Rv2_102213 vanilla_v24b.gms) to $\mathrm{C} / \mathrm{C}++$ to more seamlessly integrate with the Borg-MOEA software. We tested the consistency of our port with the original GAMS code (Online Resource Fig. ESM1) and found that results from our port are virtually indistinguishable from those of the original GAMS code for the deterministic optimal solution with default parameters. The ported DICE model (CDICE2013) along with the coupled DOECLIM climate model (discussed below) are available at https://github.com/scrim-network/cdice_doeclim.

\subsection{Representing key aspects of uncertainty in DICE}

We represent a key uncertainty in DICE in order to calculate the expectations and reliabilities associated with each of the objectives. To do so, we produce 100 samples from a log-normal fit to a recently published distribution of climate sensitivity (Olson et al. 2012) $(\mu=1.098001424$, $\sigma=0.265206276$ ) using inverse-transform sampling.

To improve the consistency of the model temperature hindcasts with historical temperature records, we couple the DOECLIM climate model (Kriegler 2005; Goes et al. 2011) to CDICE2013. DOECLIM is a simple energy-balance model that connects the troposphere and upper ocean with a diffusive deep-ocean layer. We calibrate the climate sensitivity, ocean diffusivity, and aerosol forcing parameters in DOECLIM using the National Aeronautics and Space Administration Goddard Institute for Space Studies (NASA-GISS) atmospheric temperature anomalies and radiative forcing data (Hansen et al. 2010) (Online Resource 
Fig. ESM2) from years 1900 through 2010. We calibrate the parameters by iterating over values of climate sensitivity while optimizing the ocean diffusivity and aerosol forcing parameters that minimizes the sum of square residuals between the model results and NASA-GISS temperature data (Online Resource Fig. ESM3). We acknowledge that using a Markov-Chain Monte Carlo analysis to obtain the full joint distributions of the three parameters would be a more formal and exhaustive calibration, but our approximate method seems sufficient for the purposes of this study.

A climate sensitivity sample with the calibrated ocean diffusivity and aerosol forcing parameters represent a single state of the world (SOW). For each SOW, a hindcast of atmospheric temperature anomalies is produced and used as the initial condition for the CDICE2013 projections (Online Resource Fig. ESM3). For every evaluation of DICE in the optimization process, the endogenous variables are recalculated for each state of the world, producing 100 values or time-series of each endogenous variable.

Coupling DOECLIM to CDICE2013 not only helps maintain the relationship among model parameters throughout the SOW sampling process, but shows that a better understanding of the science while using past (hindcast) information pushes the Pareto-optimal solution set closer to the ideal solution point (Online Resource Fig. ESM4). This improvement in objective space translates to possible additional satisficing solutions in decision space and thus an improved compromise across the preferences of the stakeholders.

\subsection{Defining objectives}

We define four objective functions.

$$
\begin{gathered}
E U M=n_{\text {sow }}{ }^{-1} \Sigma_{i} W_{i} \\
R E L 2 C=n_{\text {sow }}{ }^{-1} \Sigma_{i}\left\{\max _{t}\left(T_{A T, t}\right) \leq 2.0\right\}_{i} \\
\mathrm{ABATE}=n_{\text {sow }}{ }^{-1} \Sigma_{i} \Sigma_{t} \Lambda_{t, i}\left(1+r i_{t, i}\right)^{-t} \\
D A M=n_{\text {sow }}{ }^{-1} \Sigma_{i} \Sigma_{t} \Omega_{t, i}\left(1+r i_{t, i}\right)^{-t}
\end{gathered}
$$

Where $n_{\text {SOW }}$ is the number of SOW and $i$ is an index over each SOW. The EUM objective is similar to that defined in the original DICE model, except that we calculate the expectation of utility over the states of the world previously described. The REL2C objective is the fraction of SOW where the maximum deviation in atmospheric temperature $\left(\mathrm{T}_{\mathrm{AT}}\right)$ at any time in the model projection $t$ remains at or below 2.0 degrees Celsius. The expression $\max _{t}\left(T_{A T, t}\right) \leq 2.0$ produces one if true or zero if false for SOW $i$. The ABATE and DAM objectives are the expectations over the SOW of the net-present value of the abatement costs $(\Lambda)$ and climate damages $(\Omega)$, respectively, summed over time. The variable $r i$ is the real interest rate calculated endogenously within the model and is a function of time $t$ and SOW $i$. The EUM (Eq. 1) and REL2C (Eq. 2) objectives are maximized while the ABATE (Eq. 3) and DAM (Eq. 4) objectives are minimized. 


\subsection{Optimization}

We use the Borg Multi-Objective Evolutionary Algorithm (Borg-MOEA) to search for the complex Pareto surface generated by the four-objective functions used in our problem formulation (Hadka and Reed 2012a). Borg-MOEA is an advanced evolutionary optimization algorithm and has been shown to be one of the most powerful optimization algorithms to date (Hadka and Reed 2012b; Woodruff et al. 2013). We chose Borg-MOEA as our optimization algorithm for its efficiency and reliability in finding the complex four-dimensional Pareto surface associated with our problem formulation.

The algorithm uses $\varepsilon$-dominance (Laumanns et al. 2002) as a means of numerical precision in objective space. We use epsilon values of $0.1,0.01,0.05$, and 0.05 for the EUM, REL2C, ABATE, and DAM objectives respectively. This yields 2914 solutions (2251 solutions in the inertia constrained formulation) that create the four-dimensional Pareto-optimal surface.

We define convergence to the Pareto-optimal surface through the use of the Borg-MOEA operator selection probabilities and Pareto-improvements during the optimization (Online Resource Fig. ESM5, ESM6). Borg-MOEA uses a combination of operators that select the next potential solutions to be tested. Once the uniform mutation operator (UM) takes over the solution proposition process (i.e. high probability) and the Pareto-improvement rate drops to $1 \%$ or less of the size of the solution set, additional optimization time would yield only marginal gains in solution quality.

Acknowledgements We thank D. Hadka for his technical support with the Borg-MOEA. We also thank W. Nordhaus, D. Diaz, C. and B. Forest, N. Tuana, M. Adler, D. Anthoff, M. Oppenheimer, R. Lempert, V. Bosetti, and the anonymous reviewers for invaluable input. This work was partially supported by the National Science Foundation (NSF) through the Network for Sustainable Climate Risk Management (SCRiM) under NSF cooperative agreement GEO-1240507 and the Penn State Center for Climate Risk Management. Any opinions, findings, and conclusions or recommendations expressed in this material are those of the author(s) and do not necessarily reflect the views of the NSF.

Open Access This article is distributed under the terms of the Creative Commons Attribution 4.0 International License (http://creativecommons.org/licenses/by/4.0/), which permits unrestricted use, distribution, and reproduction in any medium, provided you give appropriate credit to the original author(s) and the source, provide a link to the Creative Commons license, and indicate if changes were made.

\section{References}

Anthoff D, Tol RSJ (2009) The impact of climate change on the balanced growth equivalent: An application of FUND. Environ Resour Econ 43:351-367. doi:10.1007/s10640-009-9269-5

Banzhaf HS (2009) Objective or Multi-Objective? Two Historically Competing Visions for Benefit-Cost Analysis. Land Econ 85:3-23. doi:10.3368/le.85.1.3

Bentham J (1879) An Introduction to the Principles of Morals and Legislation. Clarendon Press, Oxford

Butler MP, Reed PM, Fisher-Vanden K, et al. (2014) Identifying parametric controls and dependencies in integrated assessment models using global sensitivity analysis. Environ Model Softw 59:10-29. doi:10. 1016/j.envsoft.2014.05.001

Drouet L, Bosetti V, Tavoni M (2015) Selection of climate policies under the uncertainties in the Fifth Assessment Report of the IPCC. Nat Clim Chang 5:937-940. doi:10.1038/nclimate2721

Ellsberg D (1961) Risk, Ambiguity, and the Savage Axioms. Q J Econ 75:643-669

Goes M, Tuana N, Keller K (2011) The economics (or lack thereof) of aerosol geoengineering. Clim Change 109:719-744. doi:10.1007/s10584-010-9961-Z 
Greenstone M, Kopits E, Wolverton A (2013) Developing a social cost of carbon for us regulatory analysis: A methodology and interpretation. Rev Environ Econ Policy 7:23-46. doi:10.1093/reep/res015

Grübler A, Nakićenović N, Victor DG (1999) Dynamics of energy technologies and global change. Energy Policy 27:247-280. doi:10.1016/S0301-4215(98)00067-6

Gupta S, Tirpak DA, Burger N, et al. (2007) Policies, Instruments and Co-operative Arrangements. In: Metz B, Davidson OR, Bosch PR, et al. (eds) Climate Change 2007: Mitigation. Contribution of Working Group III to the Fourth Assessment Report of the Intergovernmental Panel on Climate Change. Cambridge University Press, Cambridge, United Kingdom and New York, NY, USA, pp. 745-807

Hadka D, Reed P (2012a) Borg: An Auto-Adaptive Many-Objective Evolutionary Computing Framework. Evol Comput 21:1-30. doi:10.1162/EVCO_a_00075

Hadka D, Reed P (2012b) Diagnostic Assessment of Search Controls and Failure Modes in Many-Objective Evolutionary Optimization. Evol Comput 20:423-452. doi:10.1162/EVCO_a_00053

Hall JW, Lempert RJ, Keller K, Hackbarth A, Mijere C, McInerney DJ (2012) Robust Climate Policies Under Uncertainty: A Comparison of Robust Decision Making and Info-Gap Methods. Risk Analysis 32(10):16571672. doi:10.1111/j.1539-6924.2012.01802.x

Hansen J, Ruedy R, Sato M, Lo K (2010) Global surface temperature change. Rev Geophys 48:RG4004. doi:10. 1029/2010RG000345

Heggelund G (2007) China's Climate Change Policy: Domestic and International Developments. Asian Perspect 31:155-191

Held H, Kriegler E, Lessmann K, Edenhofer O (2009) Efficient climate policies under technology and climate uncertainty. Energy Econ 31:S50-S61. doi:10.1016/j.eneco.2008.12.012

Herman JD, Zeff HB, Reed PM, Characklis GW (2014) Beyond Optimality: Multistakeholder Robustness Tradeoffs for Regional Water Portfolio Planning Under Deep Uncertainty. Water Resour Res 7692-7713. doi:10.1002/2014WR015338

Keller K, Tan K, Morel FMM, Bradford DF (2000) Preserving the ocean circulation: Implications for climate policy. Climatic Change 47(1-2):17-43

Keller K, Hall M, Kim S-R, Bradford DF, Oppenheimer M (2005) Avoiding dangerous anthropogenic interference with the climate system. Climatic Change 73:227-238

Keller K, McInerney D, Bradford DF (2008) Carbon dioxide sequestration: How much and when? Clim Change 88:267-291. doi:10.1007/s10584-008-9417-x

Kolstad C, Toman M (2001) The economics of climate policy. Discussion Paper 00-40REV, Resources for the Future, Washington, DC

Kriegler E (2005) Imprecise Probability Analysis for Integrated Assessment of Climate Change. Dissertation, Universitat Potsdam

Laumanns M, Thiele L, Deb K, Zitzler E (2002) Combining convergence and diversity in evolutionary multiobjective optimization. Evolutionary Computation 10(3):263-282

McGranahan G, Balk D, Anderson B (2007) The rising tide: assessing the risks of climate change and human settlements in low elevation coastal zones. Environ Urban 19:17-37. doi:10.1177/0956247807076960

McInerney D, Lempert R, Keller K (2012) What are robust strategies in the face of uncertain climate threshold responses? Clim Change 112:547-568. doi:10.1007/s10584-011-0377-1

National Research Council (2009) Informing Decisions in a Changing Climate. The National Academies Press, Washington, DC

Nordhaus WD (2013) The climate casino: Risk, uncertainty, and economics for a warming world. Yale University Press, New Haven

Olson R, Sriver R, Goes M, et al. (2012) A climate sensitivity estimate using Bayesian fusion of instrumental observations and an Earth System model. J Geophys Res Atmos 117:1-11. doi:10.1029/2011JD016620

Ramsey FP (1931) Foundations Essays in Philosophy, Logic, Mathematics, and Economics. Humanities Press, Atlantic Highlands, New Jersey, USA

Rawls J (2001) The Law of Peoples: With, The Idea of Public Reason Revisited. Harvard University Press, Cambridge

Simon H (1955) A Behavioral Model of Rational Choice. Q J Econ 69:99-118. doi:10.2307/1884852

United Nations (1992) The United Nations Framework Convention on Climate Change. (FCCC/INFORMAL/84 GE.05-62220, Article 2; http://unfccc.int/files/essential_background/background_publications_htmlpdf/ application/pdf/conveng.pdf)

U.S. Office of Management and Budget (2003) Management's Responsibility for Internal Control (Circular A-4; https://www.whitehouse.gov/omb/circulars_a004_a-4/)

Von Neumann J, Morgenstern O (1945) Theory of games and economic behavior. Bull Amer Math Soc 51(7): 498-504

Weyant J, Davidson O, Dowlatabadi H, et al. (1996) Integrated Assessment of Climate Change: An Overview and Comparison of Approaches and Results. In: Bruce JP, Yi H, Haites EF (eds) Climate Change 1995: 
Economic and Social Dimensions of Climate Change: Contribution of Working Group III to the Second Assessment Report of the Intergovernmental Panel on Climate Change. Cambridge University Press, Cambridge, UK, pp. 371-393

Woodruff MJ, Reed PM, Simpson TW (2013) Many objective visual analytics: Rethinking the design of complex engineered systems. Struct Multidiscip Optim 48:201-219. doi:10.1007/s00158-013-0891-z

Yohe G (2009) Toward an integrated framework derived from a risk-management approach to climate change. Clim Change 95:325-339. doi:10.1007/s10584-009-9614-2 